\title{
LOWER FRATTINI SERIES IN FINITELY GENERATED SOLUBLE GROUPS
}

\author{
JOHN C. LENNOX AND JAMES WIEGOLD
}

\begin{abstract}
It is proved that some term of the lower Frattini series of a finitely generated abelian-by-polycyclic group is trivial. The analogous result for finitely generated centre-by-metabelian groups is false.
\end{abstract}

1. Introduction. We define the lower Frattini series

$$
G=\Phi_{0}(G) \geqslant \Phi_{1}(G) \geqslant \cdots \geqslant \Phi_{n}(G) \geqslant \cdots
$$

of a group $G$ in the obvious manner: $\Phi_{n}(G)$ is the Frattini subgroup $\Phi\left(\Phi_{n-1}(G)\right)$ of its predecessor for each $n \geqslant 1$. The aim of this note is to set up an $A N$-dichotomy (in the sense of P. Hall [2]) for finitely generated soluble groups, based on the termination or otherwise of this series in 1. In fact we prove a little more than is strictly necessary for establishing this dichotomy; it would be enough to prove the first part of the following theorem for abelianby-nilpotent groups:

THEOREM. (i) For every finitely generated abelian-by-polycyclic group $G$, there exists an $r$ such that $\Phi_{r}(G)=1$.

(ii) There exists a two generator centre-by-metabelian group $W$ whose centre $Z$ is nontrivial and such that $\Phi_{2}(W)=\Phi_{1}(W)=Z$.

The proof of (i) depends on two lemmas of P. Hall [2] describing the structure of the abelian group at the bottom of a finitely generated abelian-bypolycyclic group, and on some simple auxiliary results (\$2). As for the example, of which there are very many, perhaps the most interesting is one based on results of $P$. Hall in [1]. It is a two-generater group $W$ with centre $Z$ any prescribed countable divisible abelian group with $W / Z$ isomorphic to the wreath product of two infinite cyclic groups. Clearly $\Phi_{1}(W)=Z=\Phi_{2}(W)$.

It will be readily seen that the proof of (i) works equally well for subgroups of the big group.

We thank the referee for some useful comments.

2. Notation and auxiliary results. We use the usual left norming convention for commutators. The lower central series of a group $G$ is

$$
G=\gamma_{1}(G) \geqslant \gamma_{2}(G)=G^{\prime} \geqslant \cdots \geqslant \gamma_{n}(G) \geqslant \cdots .
$$

Other notation is standard.

Received by the editors June 20, 1974 and, in revised form, October 9, 1974.

AMS (MOS) subject classifications (1970). Primary 20E15, $20 \mathrm{E} 20$.

Key words and phrases. Frattini subgroup, finitely generated soluble group.

○ American Mathematical Society 1976 
LEMMA 2.1. Let $G$ be a nilpotent group, $H$ a subgroup of $G$ and $N$ a normal subgroup of $G$. Then $\Phi_{r}(G) \geqslant \Phi_{r}(H)$ and $\Phi_{r}(G / N) \geqslant \Phi_{r}(G) N / N$ for all $r$.

Proof. Both parts follow from the well-known fact that $\Phi(X)=\bigcap X^{\prime} X^{p}$ for a nilpotent group $X$, where the intersection is taken over all primes $p$.

LEMMA 2.2. Let $G$ be a finitely generated nilpotent group of class $c$. Then $\gamma_{c}(\Phi(G))$ is finite.

Proof. The result is clear for abelian groups: the Frattini subgroup is finite in that case. So look at the case of general $c$. Observe first that $\Phi(G) \leqslant G^{\prime} G^{p}$ for every prime $p$, so that $\gamma_{c}(\Phi(G))$ is in the subgroup generated by commutators of the form $d=\left[x_{1}, \ldots, x_{c}\right]$, with all $x_{i}$ in $G^{\prime} G^{p}$. Since all commutators of weight $c$ are central, $d$ is a product of commutators like $\left[y_{1}^{p}, \ldots, y_{c}^{p}\right]$

$=\left[y_{1}, \ldots, y_{c}\right]^{p^{c}}$, which is certainly in $\gamma_{c}(G)^{p}$. Thus $\gamma_{c}(\Phi(G)) \leqslant \cap \gamma_{c}(G)^{p}$; this intersection is $\Phi\left(\gamma_{c}(G)\right)$ since $\gamma_{c}(G)$ is abelian. Thus $\gamma_{c}(\Phi(G))$ is finite since $\gamma_{c}(G)$ is finitely generated abelian.

COROLlaRY 2.3. If $G$ is a finitely generated nilpotent group, then $\Phi_{r}(G)=1$ for some $r$.

Proof. This is obvious for abelian $G$. If $G$ is of class $c>1$, then $\Phi(G)$ is finite-by-class $(c-1)$ by Lemma 2.2 , so that the obvious induction assumption and Lemma 2.1 together give that $\Phi_{s}(G)$ is finite for some $s$. Thus by Lemma 2.1 again, $\Phi_{t}\left(\Phi_{s}(G)\right)=1$ for some $t$, so that $\Phi_{s+t}(G)=1$.

3. Proof of the theorem. We have already dealt with part (ii) in the Introduction. Let $G$ be a finitely generated group having an abelian normal subgroup $A$ such that $G / A$ is polycyclic. By [3], $\Phi(G / A)$ is nilpotent, while $\Phi(G / A) \geqslant \Phi(G) A / A$ for evident and well-known reasons. Using Lemma 2.1 repeatedly, it is routine to verify that $\Phi_{n}(G / A) \geqslant \Phi_{n}(G) A / A$ for all $n$; applying Corollary 2.3 to the finitely generated nilpotent group $\Phi(G / A)$, we see that $\Phi_{n}(G) \leqslant A$ for some $n$. But $A$ is a $B$-group in the sense of Hall [2]; and by Lemmas 8 and 12 of that paper, $\Phi(A)$ is of finite exponent. Since $A$ is abelian, $\Phi_{n+1}(G) \leqslant \Phi(A)$ so that $\Phi_{n+1}(G)$ is of finite exponent. But it is clear that the lower Frattini series of an abelian group of finite exponent hits 1 after finitely many steps (after $\lambda$ steps in fact, where $\lambda$ is the highest power of any prime dividing the exponent). Thus $\Phi_{n+\lambda+1}(G)=1$, and the proof of part (i) is complete.

\section{REFERENCES}

1. P. Hall, Finiteness conditions for soluble groups, Proc. London Math. Soc. (3) 4 (1954), 419-436. MR 17, 344.

2. 595-622. MR 22 \#1618.

3. K. A. Hirsch, On infinite soluble groups. V, J, London Math. Soc. 29 (1954), 250-251. MR 15,851 .

Department of Pure Mathematics, University College, P. O. Box 78, Cardiff CF1 1XL, WALES 